\title{
Wagerer, Wolfgang (2019). Zum Hören ermutigen. Identitätskonstruktionen in Begegnung mit biblischen Texten (Religionspädagogik innovativ 31). Stuttgart: Kohlhammer. ISBN 978-3-I 7-034890-5. 348 Seiten.
}

\section{Kristin Konrad}

Institut für Lehrerfortbildung Essen/Gymnasium Augustinianum Greven i.Westf (k.konrad@ifl-fortbildung.de)

\begin{abstract}
„Höre, Israel!“ (Dtn 6,4): Eine grundlegende Botschaft biblischer Texte ist die, aufzumerken, zuzuhören, auf die Tora zu hören (171-176). Im christlichen Kontext - und aus christlicher Perspektive spricht der Autor - ist die Liturgie ein zentraler Ort, an dem Bibel präsent ist (30f.). In liturgischen Feiern wird die biblische Lesung als Hörerlebnis inszeniert. Somit liegt es nahe, das Hören als Wahrnehmung und als Prozess der individuellen Konstruktion von Sinn und Verstehen auch für schulischen Unterricht zu beleuchten.
\end{abstract}

Belesen und erfahrungsstark widmet sich Wolfgang Wagerer diesem Bedarf. Nach einem stark autobiografisch geprägten Durchgang durch bibeldidaktische Konzepte der letzten Jahrzehnte im deutschsprachigen Raum (19-28) ermittelt der Autor das ,Elementare', die grundlegende Bedeutung des Hörens und auch des Erzählens z. B. in der (Entwicklungs-)Psychologie (49-67). Es geht immer wieder um das Hören selbst, aber damit verknüpft auch um die Bedeutung von Narrativität für menschliche Existenz. Wagerer wirft einen poetologischen Blick (78) auf exemplarische literarische Texte, lässt Schriftsteller*innen zu Wort kommen und führt seine Überlegungen immer wieder zur Theologie zurück. Auf diese Weise sucht er Anschlussstellen für bibeldidaktische Überlegungen. Auch den Bereich der Identitätssuche (97-118) befragt Wagerer auf die Bedeutung des Hörens und Erzählens hin und kommt zu dem Schluss, es sei angebracht, „,eine Didaktik des Hörens aus der auditiven Konstruktion von Identität zu entwerfen“ (118). Diese konkretisiert er im Anschluss (118-123), vornehmlich im Dialog mit der Bibeldidaktik von Maurice Baumann. Wagerer greift insgesamt auf einen sehr breiten theoretischen Horizont zurück. Eine stärkere Gewichtung und Strukturierung würde einen leserfreundlichen Akzent setzen, z. B. durch eine Zusammenführung der bibeldidaktischen Impulse der einzelnen Kapitel. Weitergeführt werden sie durchaus, was beispielsweise an den Bemerkungen zu Leerstellen in Literatur (mit Hilde Domin, 96) und Exegese (mit Iser, 220) deutlich wird. Spannend wäre zudem in diesem Kontext ein inklusiver Contra-Punkt, der die Perspektive von Gehörlosen oder aber von Kindern und Jugendlichen mit wenig Deutschkenntnissen in den Blick nimmt - entwicklungspsychologisch, biblisch-hermeneutisch und in Bezug auf didaktische Konkretionen.

Der Rekurs auf die biblische Perspektive des Hörens erfolgt insgesamt mehrfach: kurz, aber inhaltlich breit angelegt über „,identitätsstiftendes Hören in der Bibel“ (124) oder biblische Inszenierungen des Hörens (171-176), dann über theologische Schwerpunktsetzungen zum Buch Exodus (303-313), also anhand einer Tiefenbohrung in der zentralen biblischen Erzählung der Untersuchung. Die biblische Rückbindung ist somit deutlich sichtbar und erdet die Darstellung in Bezug auf ihren Lerngegenstand, biblische Texte.

Der praktisch-didaktische Ertrag findet sich im vorletzten Kapitel: Hier entwickelt der Autor beispielhaft eine Audende, also „das zu Hörende“ (177). Audende meint hier konkret den Grundriss für einen Hörtext, beispielhaft vollzogen für das gesamte Buch Exodus, ausgelegt auf unterrichtliche Zwecke (292-302). Wagerer nähert sich der Audende Schritt für Schritt an. Dies geschieht erstens über die Frage nach dem ,Wie' des Erzählens (140-184), auf verschiedenen Ebenen beleuchtet, so z. B. in Bezug auf Gesprächsformen im Unterricht oder auf die Bewahrung der Fremdheit der biblischen Texte. Hier stellt Wagerer mit Horst Klaus Berg fest, dass die Defizite des Bibelunterrichts nicht im methodischen, sondern im hermeneutischen Feld zu verorten seien (166). Zweitens setzt der Autor literaturtheoretische Schwerpunkte und klärt so sein analytisches Handwerkszeug für die Bibeltexte (185-223). Aus der Leserperspektive finden sich kleine Unklarheiten: Im Schaubild „Strukturanaloges Erzählen - Audende“ (185) wird z. B. der Erzählbogen dem biblischen Buch zugeordnet. Im folgenden Text versteht man den Erzählbogen jedoch als didaktisches Konstrukt, das deutlich auf die tatsächliche Zielgruppe hin modelliert ist (186). Somit wird nicht ganz deutlich, ob der didaktische oder der literarische Erzählbogen, die bereits differenziert wurden (151), gemeint ist. Kurz gesagt: Als Leserin ist man ob der Vielfalt der Konzepte im Hintergrund und Terminologie beeindruckt, sie kann aber auch verwirren. 
Eine dritte Annäherung an die Audende bietet der strukturierte Überblick über wichtige Etappen der wissenschaftlichen Exegese des Buches Exodus der letzten Jahrzehnte (Exkurs, 224-240). Der Darstellung mangelt es wieder nicht an Vielseitigkeit. Hervorzuheben ist Wagerers Wertschätzung z. B. der feministischen Theologie (236), die sich in aktuellen theologischen Debatten jedoch mittlerweile deutlich kontextbezogener aufstellt (postkoloniale Zugänge, z. B. Gale A. Yee, Musa W. Dube). Von der exegetischen Darstellung her legt Wagerer das Buch Exodus überblicksartig aus (241-292). Er stellt Leitworte (z. B. befreien und vertreiben; herausführen, 278) heraus, um der Audende Struktur zu verleihen und den Hörer*innen Orientierung zu bieten (277). An dieser Stelle wird exemplarisch deutlich, wie ein gutes exegetisches Fundament ein didaktisches Erschließen strukturiert und ihm theologische Qualität verleiht. Wagerer öffnet seine Exegese nach der Erstellung der Audende schließlich noch einmal mit Hilfe der theologischen Schwerpunktsetzungen des Schlusskapitels (303-313). Er nimmt ihr so die Starrheit einer ,Musterlösung', hält sie auch von seinem Selbstanspruch her flexibel für verschiedene Kontexte und diverse Adressat*innen.

Zum Schluss betont der Autor, dass Kinderbibeln als Hilfe für eine Audende wenig böten und dass die Rolle der Lehrer*innen und ihre biblisch-theologische Kompetenz eminent wichtig seien (313-321). Letzteres war bereits deutlich geworden; ergänzend ließen sich Desiderate für die Aus-, Fort- und Weiterbildung von Religionslehrer*innen entwickeln.

Wagerer spannt einen weiten Bogen von hermeneutischer Grundlegung hin zu didaktischer Konkretion. Er lässt die Leser*innen in beiden Bereichen von seinen umfassenden Kenntnissen und Erfahrungen profitieren. Als Lehrerin wünscht man sich, wie von manchem Band der Reihe ,Religionspädagogik innovativ', einen Folgeband in Form einer Unterrichtshilfe: mit Basics zur Begründung des biblisch-hermeneutischen und didaktischen Schwerpunkts auf dem Hören, mit dem theologisch klaren Konzentrat zum Exodusbuch des letzten Kapitels und einer Anleitung zum Erstellen einer Audende, die hier beispielhaft durchgeführt wurde. So ließe sich der Graben zwischen religionspädagogischer Forschung und Unterrichtspraxis situativ, aber exemplarisch überbrücken. 